\title{
BMJ Open Evaluating Maternity Units: a prospective cohort study of freestanding midwife-led primary maternity units in New Zealand - clinical outcomes
}

Celia P Grigg, ${ }^{1}$ Sally K Tracy, ${ }^{2}$ Mark Tracy, ${ }^{2}$ Rea Daellenbach, ${ }^{3}$ Mary Kensington, ${ }^{3}$ Amy Monk, ${ }^{4}$ Virginia Schmied ${ }^{5}$

To cite: Grigg CP, Tracy SK, Tracy M, et al. Evaluating Maternity Units: a prospective cohort study of freestanding midwife-led primary maternity units in New Zealandclinical outcomes. BMJ Open 2017;7:e016288. doi:10.1136/ bmjopen-2017-016288

- Prepublication history for this paper is available online. To view these files please visit the journal online (http://dx.doi. org/10.1136/bmjopen-2017016288).

Received 5 February 2017 Revised 12 May 2017 Accepted 22 May 2017

\section{(a) CrossMark}

${ }^{1}$ Division of Midwifery, School of Health Sciences, University of Nottingham, Nottingham, UK

${ }^{2}$ University of Sydney, Sydney, Australia

${ }^{3}$ Ara Institute of Canterbury, Christchurch, New Zealand ${ }^{4}$ University of Technology Sydney, Sydney, Australia ${ }^{5}$ School of Nursing and Midwifery and the Family and Community Health, University of Western Sydney, Sydney, Australia

Correspondence to

Dr Celia P Grigg;

celia.grigg@nottingham.ac.uk

\section{ABSTRACT}

Objective To compare maternal and neonatal birth outcomes and morbidities associated with the intention to give birth in a freestanding primary level midwifeled maternity unit (PMU) or tertiary level obstetric-led maternity hospital (TMH) in Canterbury, Aotearoa/New Zealand.

Design Prospective cohort study.

Participants 407 women who intended to give birth in a PMU and 285 women who intended to give birth at the TMH in 2010-2011. All of the women planning a TMH birth were 'low risk', and 29 of the PMU cohort had identified risk factors.

Primary outcomes Mode of birth, Apgar score of less than 7 at $5 \mathrm{~min}$ and neonatal unit admission. Secondary outcomes: labour onset, analgesia, blood loss, third stage of labour management, perineal trauma, nonpharmacological pain relief, neonatal resuscitation, breastfeeding, gestational age at birth, birth weight, severe morbidity and mortality.

Results Women who planned a PMU birth were significantly more likely to have a spontaneous vaginal birth $(77.9 \%$ vs62.3\%, adjusted OR (AOR) $1.61,95 \% \mathrm{Cl}$ 1.08 to 2.39 ), and significantly less likely to have an instrumental assisted vaginal birth (10.3\%vs $20.4 \%$, AOR $0.59,95 \% \mathrm{Cl} 0.37$ to 0.93 ). The emergency and elective caesarean section rates were not significantly different (emergency: PMU $11.6 \%$ vs TMH $17.5 \%$, AOR $0.88,95 \% \mathrm{Cl}$ 0.55 to 1.40 ; elective: PMU $0.7 \%$ vs TMH $2.1 \%$, AOR 0.34 , $95 \% \mathrm{Cl} 0.08$ to 1.41). There were no significant differences between the cohorts in rates of $5 \mathrm{~min}$ Apgar score of $<7$ (2.0\%vs2.1\%, AOR 0.82, 95\% Cl 0.27 to 2.52$)$ and neonatal unit admission $(5.9 \%$ vs $4.9 \%$, AOR $1.44,95 \% \mathrm{Cl}$ 0.70 to 2.96). Planning to give birth in a primary unit was associated with similar or reduced odds of intrapartum interventions and similar odds of all measured neonatal well-being indicators.

Conclusions The results of this study support freestanding midwife-led primary-level maternity units as physically safe places for well women to plan to give birth, with these women having higher rates of spontaneous vaginal births and lower rates of interventions and their associated morbidities than those who planned a tertiary hospital birth, with no differences in neonatal outcomes.
Strengths and limitations of this study

- This is the first prospective cohort study of maternal and neonatal outcomes of women who planned to give birth in freestanding primary level midwife-led units, compared with women of similar risk status who planned to give birth in a tertiary level obstetricled hospital in New Zealand.

- Participants in both cohorts received the same model of care-midwife-led continuity of carewith their own primary care midwife for antenatal, intrapartum and postpartum care, with additional specialist care as required.

- Women were not randomised to their place of birth. Selection bias was minimised by prospectively identifying women's planned place for birth antenatally at study entry and analysing the outcomes according to the place where women intended to give birth. The risk status of participants on admission in labour could not be drawn from the data source. There was no loss to follow-up.

- The seriously disruptive earthquakes, which occurred during the study period resulted in the premature end of recruitment, some disruption to birthplace choices and generalised community stress and trauma. This may have had a confounding effect on the outcomes, yet could not be controlled for in the data analysis.

\section{INTRODUCTION}

In Aotearoa/New Zealand, birthplace options include 18 secondary-level and 6 tertiary-level obstetric-led maternity hospitals (TMHs), which have midwifery and specialist obstetric, anaesthetic and paediatric services onsite; 54 freestanding primary level midwife-led maternity units (PMUs) offering birthing facilities, which have midwifery services onsite and funded home birth. ${ }^{1}$ In 2014, 87.6\% of births occurred in a secondary or tertiary hospital, $9.1 \%$ in a freestanding PMU and $3.4 \%$ were home births. ${ }^{2}$ This study examined outcomes for women who planned to give birth in the 
immediate Christchurch area, which has a TMH and four PMUs.

The maternity system has continuity of care as a core tenet, resulting in women receiving continuity of care regardless of planned or actual birthplace. ${ }^{3}$ Midwives were the primary maternity care provider (lead maternity carer) for $93.4 \%$ of women nationally, ${ }^{2}$ and all of the participants in this study. The midwives in this study continued as the primary caregiver whether the woman planned to give birth at the TMH or a PMU, changed her birthplace plan or needed specialist consultation. Consequently, the current study did not have the confounding factor of two different models of care for women in the two cohorts, so was able to compare the differences in outcomes related to planned birthplace independently of model of care.

At the time the study was proposed there were no contemporary, comprehensive systematic data addressing the important differences between the clinical outcomes achieved in freestanding primary maternity units compared with tertiary hospitals in Australia or New Zealand. The existing evidence from both Australia ${ }^{4}$ and New Zealand ${ }^{5}$ supported primary units as safe birthplaces. However, this research was limited, extremely dated and set in a context of organisation of maternity care which no longer exists (with general practitioners as the main care providers in primary units).

The contemporary international research into the comparative clinical outcomes for PMUs and obstetric-led hospitals (TMH/OU) include prospective cohort studies, ${ }^{6-8}$ a retrospective study ${ }^{9}$ and population-based cohort studies. ${ }^{10-14}$ All but three of these studies report the same pattern of similar or improved neonatal outcomes and reduced 'interventions' (such as labour augmentation, instrumental assisted birth, episiotomy and emergency caesarean section) and their associated maternal morbidity for women planning a PMU birth, when compared with women of similar well-being (risk status) planning a TMH birth. The three discrepant studies, which report worse neonatal outcomes for PMU babies than those born in an OU, are population-based studies from the USA. ${ }^{10} 12$ The lack of integration of midwifery and midwife-led maternity units (birth centres) in the American maternity system arguably limits the applicability of these, and other research from the USA, in other contemporary western contexts.

This article reports on the New Zealand arm of a larger prospective cohort study to Evaluate primary freestanding midwife-led Maternity Units (EMU) undertaken in Australia and New Zealand (2010-2012). The larger study was funded by the National Health and Medical Research Council of Australia (project grant number: 571901). The primary objective of the larger study was to compare the clinical outcomes for well ('low risk') women intending to give birth in an TMH with those for women intending to give birth in a freestanding PMU in Australia or New Zealand. Primary outcomes were mode of birth, Apgar score of less than 7 at 5 min and admission to the neonatal unit. Secondary outcomes were onset of labour, analgesia, blood loss, management of third stage of labour, perineal trauma, non-pharmacological pain relief, neonatal resuscitation, breastfeeding, gestational age at birth, birth weight, severe morbidity and mortality.

\section{METHODS}

\section{Sample and recruitment}

The study was set in the Christchurch area, in Canterbury. There is a TMH and four PMUs in the area. Two of the PMUs are located semi-rurally outside the city boundaries ( 19.6 and $35.4 \mathrm{~km}$ from the TMH), and the two urban units are situated on hospital sites but operate independently as freestanding primary maternity units $(3.8$ and $10.8 \mathrm{~km}$ from the TMH). Women who develop complications are transferred to the TMH. The TMH has a neonatal unit (NNU) which includes a tertiary level neonatal intensive care unit and lower level special care nursery.

All of the women invited to join the study had a midwife as their Lead Maternity Caregiver (LMC), who provided continuity of care from booking until 6 weeks post partum. The clients of $95 \%$ of local midwives were invited. The midwives were either members of the New Zealand College of Midwives' owned Maternity and Midwifery Provider Organisation (MMPO) $-90 \%$ of local midwives-or they had agreed to complete the forms required (17 midwives). Only 5\% of local midwives, who were not members of the MMPO, did not agree to complete the forms required. All eligible women booked to give birth in any one of the four PMUs were invited to participate. Women who booked into the tertiary hospital were invited, if they were well (at 'low risk' of pregnancy complications) based on information on the hospital booking form. An a priori decision was made to invite all women booked to give birth in any one of the PMUs to participate, regardless of the presence of risk factors. For the purposes of this study, women with 'risk factors' were defined as those with any level two or three referral criteria as defined in the New Zealand Maternity Referral Guidelines. ${ }^{3}$ For example, women who had had a previous caesarean section or were expecting twins were ineligible. Eligible women were invited to participate via a postal invitation to join the study, with a phone follow-up to those who did not respond. After 6 months of recruitment, ethics approval was sought and granted to change the study protocol and extend the follow-up calls from only PMU booked to both PMU-booked and TMH-booked eligible women, when it became evident that they were an effective recruitment tool. The recruitment rates for each group then became similar for the two cohorts. Women were able to join the study any time after hospital booking and before labour.

Recruitment began in March 2010, was suspended for 1 month after a major earthquake in September 2010 and stopped prematurely after a subsequent severe earthquake in February 2011. Following the September earthquake, two PMUs were closed for a week and 


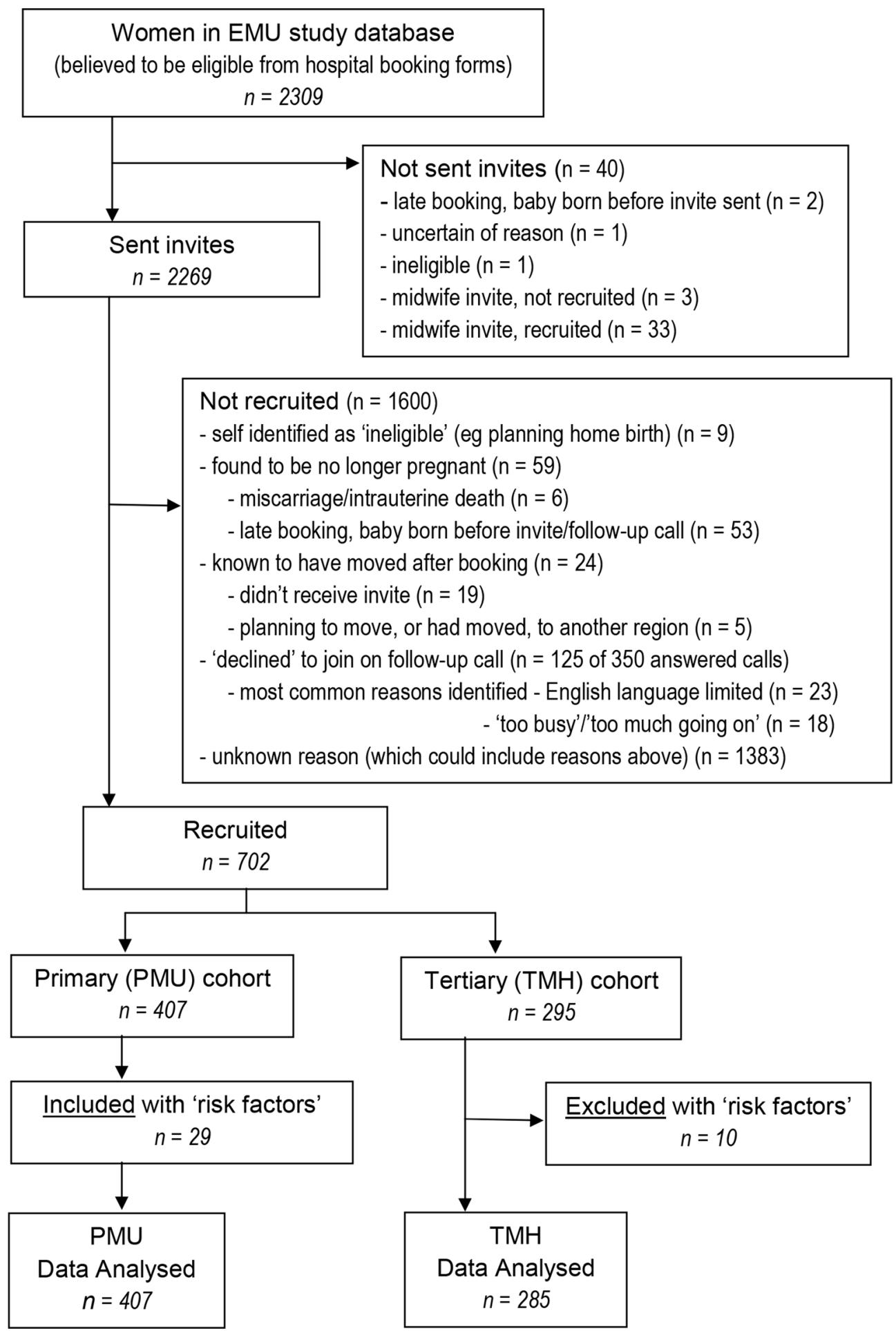

Figure 1 Flow chart of EMU study recruitment, inclusions and exclusions. EMU, Evaluate primary freestanding midwife-led Maternity Units; PMU, primary level midwife-led maternity unit; $\mathrm{TMH}$, tertiary level obstetric-led maternity hospital.

services resumed within 2 weeks. After the February earthquake, the city's busiest PMU closed and was subsequently demolished, and another was closed for 7 weeks. The semi-rural PMUs remained open. The TMH was also affected through damage to its water supply and drainage systems but remained open. Participants gave birth between March 2010 and August 2011, with 39\% giving birth before the first earthquake, $41 \%$ between the two major quakes and 20\% after the February earthquake. Figure 1 details the study recruitment, inclusions and exclusions details.

\section{Data collection}

The clinical outcome data for $88 \%$ of participants were collected from data submitted by midwives to the MMPO. Participants' midwives also completed the 'Midwives 
Transfer Form' to collect some data not captured by the MMPO database. The remaining $12 \%$ of data were provided by 17 midwives, who were not members of the MMPO, on customised study forms. Data collected (including that from the 6-week postpartum survey) were manually checked for completeness and accuracy, with incomplete or inconsistent records followed-up with the MMPO database staff, the participants' midwives or hospital records.

\section{Data analysis}

Women's outcomes were analysed by stated intention to give birth either in a PMU or TMH at the time of study entry. Measures of categorical data were analysed with $\chi^{2}$ tests and continuous data were analysed using t-test. Fisher's exact test was used with cell size $<5$ (need for neonatal resuscitation variable). Independent variables known from prior studies to be associated with the outcome of interest were examined. Those variables found to be associated in the univariate analysis with $\mathrm{p}<0.05$ were then examined in multivariate models. ORs with $95 \%$ CIs were calculated for the primary and secondary outcomes. Multivariate logistic regression was used for dichotomous outcomes to adjust for relevant confounders. Likelihood ratio tests were used to assess the contribution of each independent variable nested in the full model. Adjustments for all outcomes are outlined below the tables. Multivariate regression models were restricted to subjects with no missing values. No inferential statistics were carried out on severe maternal/neonatal morbidity and mortality outcomes due to the rarity of events and small numbers involved.

\section{RESULTS}

\section{Participants}

A total of 702 women joined the study based on their intended birthplace. Overall approximately $30 \%$ of those invited joined the study; this included women who were followed-up by telephone. Figure 1 details of the study's recruitment, inclusions and exclusions. The cohort allocation was based on the intended birthplace the women identified on their study consent form. Among the 10 women excluded from the TMH cohort analysis due to their risk status, which was subsequently identified from the database, were two women having twins, three women with BMI $>40$ and three women with a 'neurological disorder' (eg, epilepsy). Among the 29 women included in the PMU cohort identified as having 'risk factors' were 15 women with body mass indices (BMIs) of 35-42, 2 with a 'neurological disorder', 7 with 'thyroid disease' and 2 with previous caesarean (and subsequent vaginal birth). While women who have had caesareans previously are not recommended for primary unit birth in New Zealand, these two women had their PMU booking accepted. Consequently, 29 PMU women with known risk factors, and no TMH women with known risk factors or complications which made them unable to book at a PMU, were included in the analysis. There was no loss to follow-up.

Approximately half $(46.9 \%)$ of the women who intended to give birth in a PMU did so, with most plan changes occurring antenatally or in labour prior to admission. Only $12.6 \%$ of those admitted to a PMU in labour were transferred to the TMH, a further $4.7 \%$ transferred between birth and 48 hours (four women, five babies), giving a total postadmission transfer rate of $17.3 \%$. Details of the rates, reasons timing, urgency and outcomes of transfers have been reported previously. ${ }^{15}$

Table 1 shows the demographic differences between the groups. There were some significant differences between the two cohorts. The women who planned to give birth in a PMU were younger, heavier, more likely to have given birth before, to be Māori and to live rurally, than the women who planned to give birth in the TMH. There were no differences in the groups' smoking or relationship status. Additionally, data on the participants' income and education levels were sought in the survey (82\% response rate) as they are not collected by the database. The TMH respondents had significantly higher incomes than those planning a PMU birth, with no significant differences in the education levels identified.

The study's primary maternal outcome measures, analysed by intended birthplace on study entry, are illustrated in table 2. After adjusting for confounding factors (maternal age, smoking status, parity, term, augmentation, induction, excludes elective caesarean section), women from the PMU cohort were significantly more likely to have a spontaneous vaginal birth $(77.9 \%$ vs $62.3 \%$, AOR 1.61, 95\% CI 1.08 to 2.39) and less likely to have an instrumental-assisted (ventouse or forceps) vaginal birth ( $10.3 \%$ vs $20.4 \%$,AOR $0.59,95 \%$ CI 0.37 to 0.93$)$. The caesarean section rate-elective $(0.7 \%$ PMU vs $2.1 \%$ $\mathrm{TMH}$ ) or emergency (11.6 PMU vs $17.5 \% \mathrm{TMH}$ )—was not significantly different between the cohorts.

Table 2 describes secondary maternal outcomes, showing the numbers, percentages and ORs by planned place of birth. The PMU cohort was significantly more likely than women from the TMH cohort to have spontaneous labour onset (66.8\% PMU vs $49.1 \% \mathrm{TMH})$, meconium liquor (18.7\% PMU vs 12.6\% TMH), no analgesia (42.4\% PMU vs $25.0 \% \mathrm{TMH}$ ) or use non-pharmacological pain relief ( $75.0 \%$ PMU vs $65.2 \%$ TMH) and have physiological management of the third stage of labour (41.8\% PMU vs $19.3 \% \mathrm{TMH})$. Women from the PMU cohort were significantly less likely to have labour augmentation (17.2\% PMU vs $28.2 \% \mathrm{TMH}$ ) or an episiotomy $(7.8 \%$ vs $19.7 \% \mathrm{TMH})$, compared with women from the TMH cohort. The cohorts had similar rates of induction, postpartum haemorrhage, other perineal trauma (table 2) and length of labour (data not shown).

The primary neonatal outcome measures are detailed in table 3 . There were no significant differences between the cohorts in rates of 5 min Apgar score of less than 7 (incidence rate of 2.0\% PMU and 2.1\% TMH AOR 0.82, 


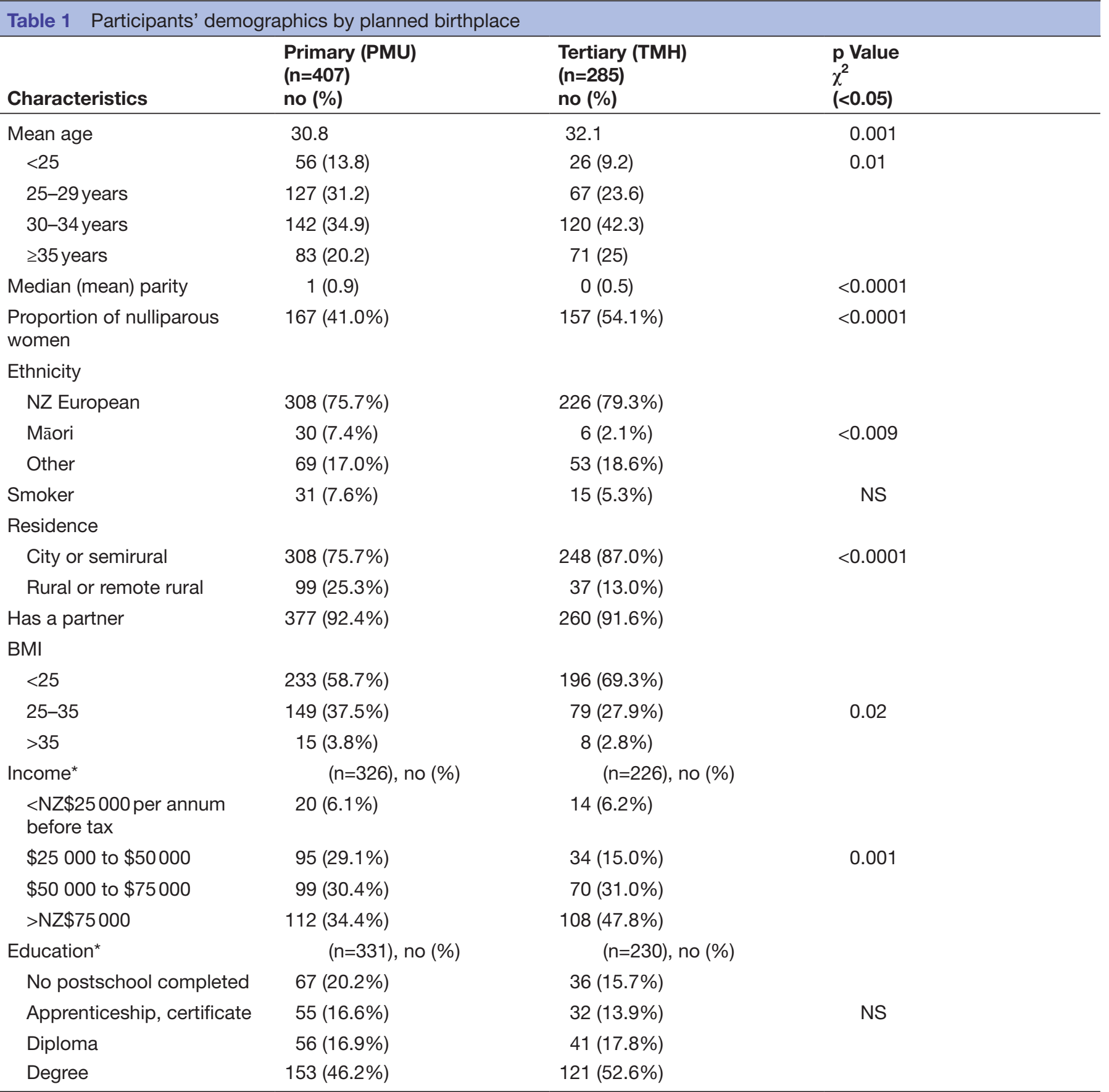

${ }^{*}$ Different denominator-survey response data, not full study cohorts (82\%).

BMI, body mass index; NS, not significant;

95\% CI 0.27 to 2.52$)$ and admission to the neonatal unit ( $5.9 \%$ vs $4.9 \%$ AOR $1.44,95 \%$ CI 0.70 to 2.96$)$. There were also no significant differences in the secondary neonatal outcomes, as detailed in table 3 .

\section{DISCUSSION}

This study found that women planning a freestanding PMU birth when compared with women of similar wellbeing (risk status) planning a TMH birth had more favourable clinical outcomes.
Studies such as this observational prospective cohort study have both limitations and strengths. The limitations include the small study size which prevented strongly powered statistical analysis of clinical outcomes and not knowing the 'risk status on admission in labour' of participants. The major disruptive earthquakes which occurred during the study period were a serious 'confounder', resulting in the premature end of recruitment; and major damage to the city and its infrastructure, including power, water, roads and hospitals. This caused generalised community stress and 
鄯部

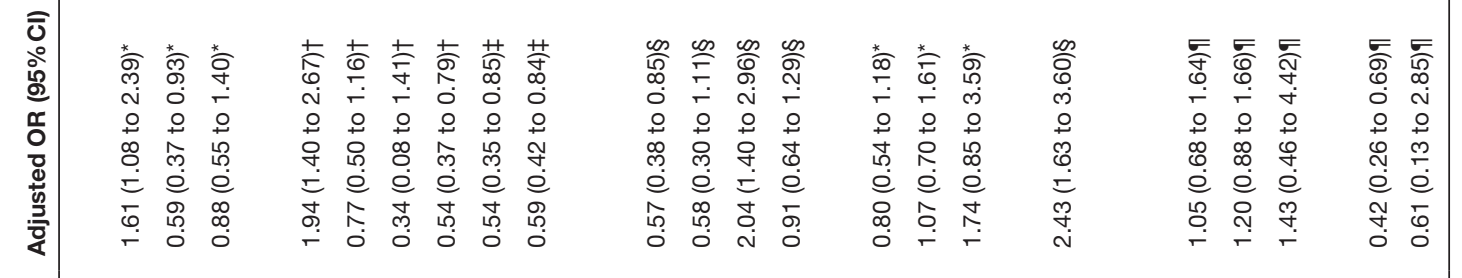

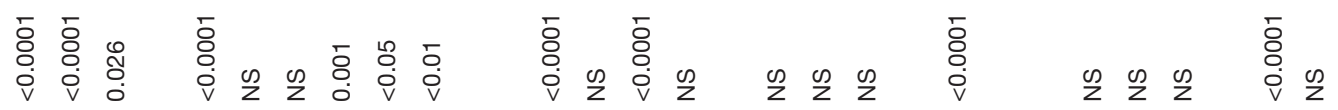

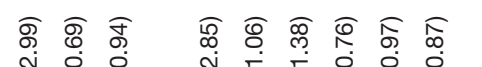

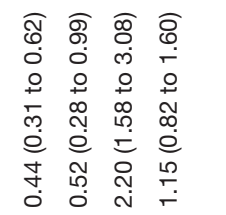

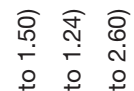

$\begin{array}{lllll}0 & 0 & 0 & 0 & 0 \\ 0 & 0 & 0\end{array}$ 莫

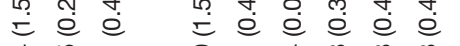

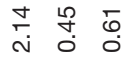

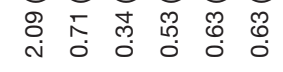

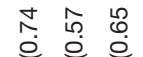
$\begin{array}{lll}0 & 0 \\ 2 & 0 & 0 \\ 0 & 0 & 0 \\ 0 & 0 & 0\end{array}$

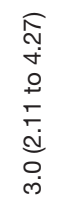

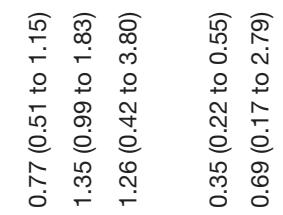

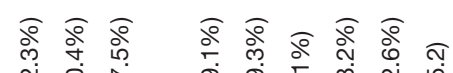

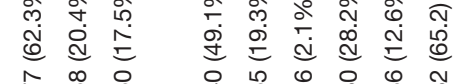

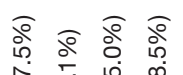

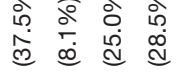

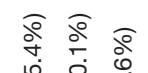

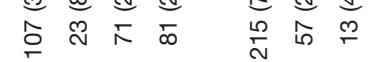

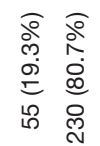

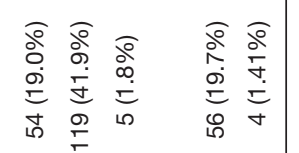

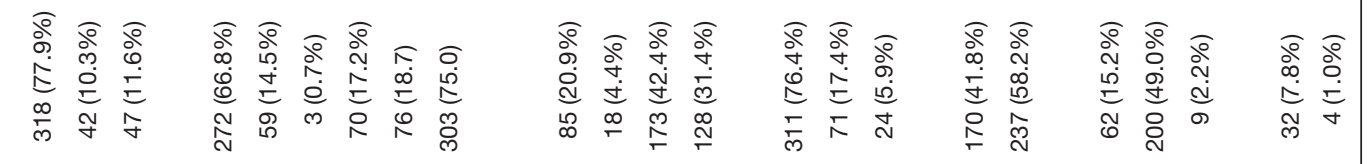

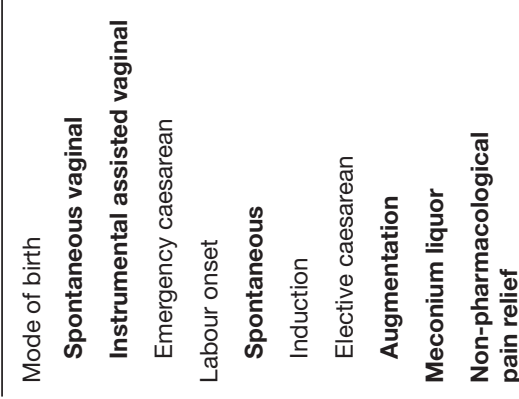

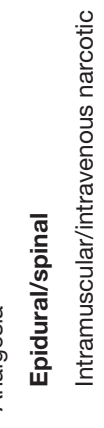

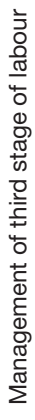

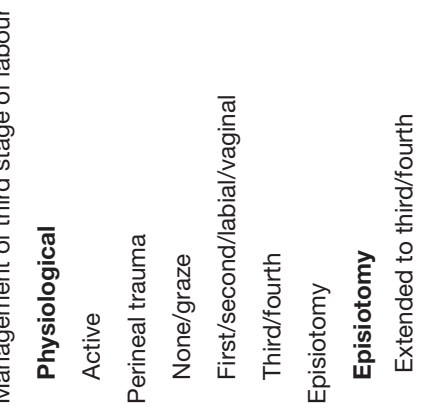


Table 3 Neonatal clinical outcomes by planned place of birth

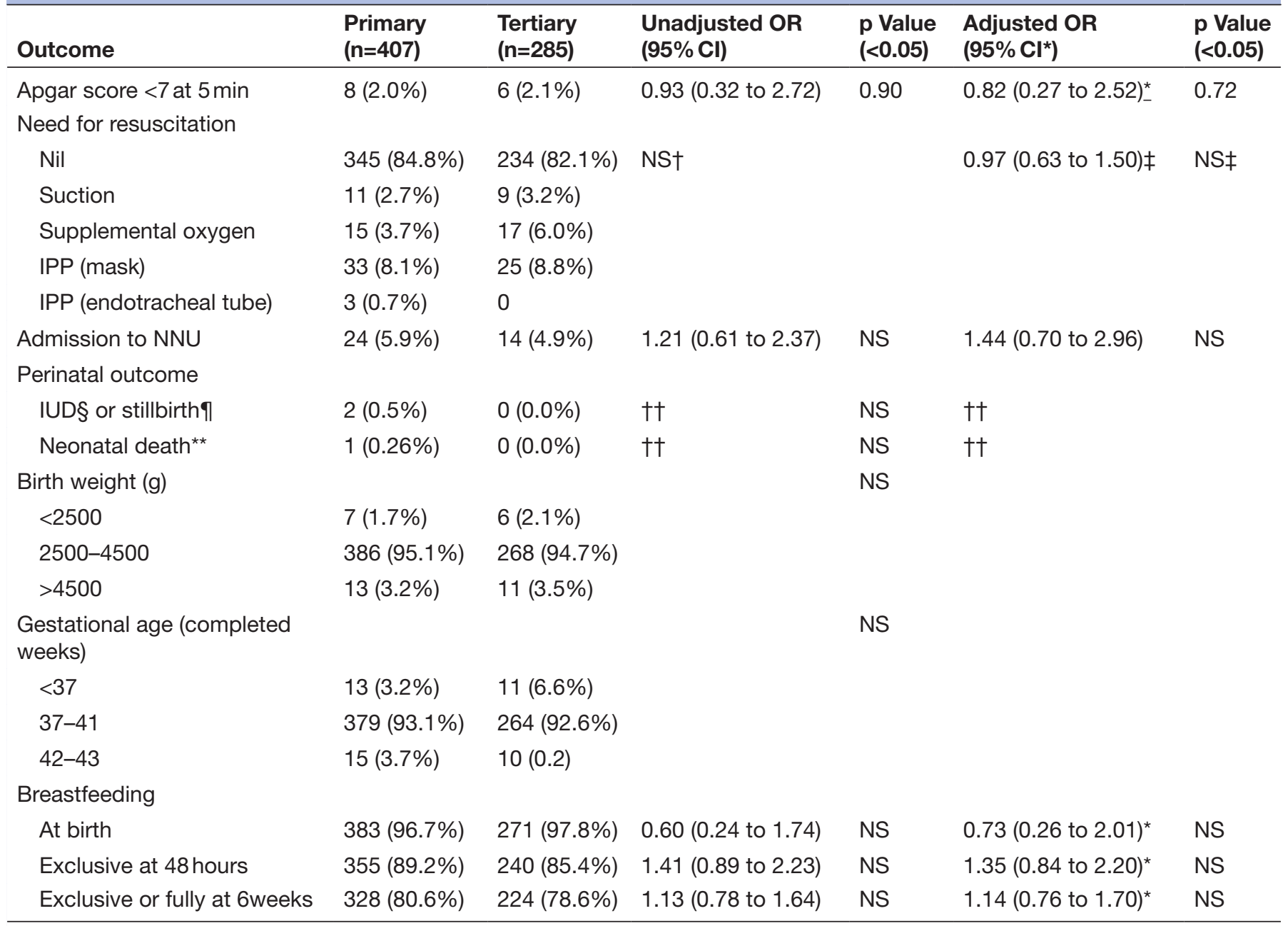

${ }^{*}$ Adjusted for maternal age, smoking status, parity, augmentation, induction, term.

†Fisher's exact test for listed proportions of modes of resuscitation.

$\ddagger$ Refers to a dichotomous outcome of 'resuscitation or not' adjusted for maternal age, smoking status, parity, augmentation, induction, term. §Antenatal IUD identified at 38 weeks during routine antenatal visit, baby slightly small for gestational age (SGA), no cause found, no pregnancy complications, well woman.

IStillbirth antepartum haemorrhage (APH)/placental abruption at 34 weeks; transfer to $\mathrm{TMH}$ and obstetric supervised care at 20 weeks with pregnancy induced hytertension $(\mathrm{PIH}) \times 3 \mathrm{a} / \mathrm{n}$ admissions.

${ }^{\star \star}$ Neonatal death at 3 weeks of age, late onset group B stretococcus (GBS) infection.

††Numbers too small. Multivariate model cannot converge.

IPP, inspiratory positive pressure; IUD, intrauterine death; NNU, neonatal unit (includes neonatal intensive care or special care nursery);

trauma and had a major confounding effect on birthplace choice (with $28 \%$ of antenatal changes in planned birthplace due to earthquakes ${ }^{16}$ ). It is not possible to quantify the quakes' impact on individual participants or identify if one cohort was more adversely affected that the other.

Another limitation is that the study sample is not representative of the childbearing women in New Zealand. The proportion of women under the age of 25 who joined the study is considerably lower than in the population of women from the region who gave birth at the time (11.9\% as compared with $21.5 \%$ of women giving birth were under 25 in the study and the Canterbury region, respectively). Māori women were also less likely to take part in the study $-5.2 \%$ of the participants and $12.5 \%$ of women birthing in Canterbury in 2010 were Māori. ${ }^{17}$ Self selection bias is also present in both groups, as all of the women chose their preferred birthplace, leaving open the possibility of psychological or motivational differences between the groups. Selecting a prospective comparative reference cohort from the referral hospital goes some way in addressing the selection bias.

Differences in the beliefs and values of the women in the PMU and TMH cohorts in this study have been identified and discussed in previous publications. ${ }^{16}{ }^{18} \mathrm{In}$ common with prior research, we found that 'safety' was the paramount consideration in women's birthplace decision-making, although the two groups had different perceptions of the concept. Accessing the specialist services/facilities (if needed) was the most important 
factor for women planning a TMH birth. ${ }^{16}$ In contrast, the PMU group identified several factors, including 'closeness to home', 'ease of access', the 'atmosphere' of the unit and avoidance of unnecessary intervention' as important. ${ }^{16}$ This study found women who planned a PMU birth expressed confidence in the birth process, their ability to give birth, the maternity system (for specialist referral or transfer) and/or the primary unit itself. ${ }^{18}$ In contrast, women who planned a TMH birth did not express confidence in these things, although almost all study participants expressed confidence in their midwife. ${ }^{18}$

The influence of the personal philosophy of midwives who choose to offer women particular birthplace options were beyond the scope of this study. In this context, LMC midwives work autonomously and independently of the birth facilities and choose their practice context, which arguably means they are not providing labour and birth care in a place in which they do not feel confident and competent. This does not mean that their practice is not influenced by the organisational context of the respective facilities, as has been demonstrated in previous New Zealand research. ${ }^{19} 20$ The focus of the current research was the women and not the 135 midwives providing their care, the majority of whom provide care in both PMU and TMH facilities and a few of whom offer only one or other option to women. The women also chose their own midwife, knowing the options she offered prior to booking with that midwife, with a few changing midwives during pregnancy, if they found the midwife's beliefs and values in conflict with their own. ${ }^{16}$ As reported previously, a relatively small proportion of study participants identified their decision was influenced by the recommendation of their midwife (PMU 4.35\%, TMH 6.24\%) via the 6 -week postpartum survey $\left(82 \%\right.$ response rate) ${ }^{16}$ Additionally, women indicated the extent to which their midwife influenced their birthplace decision on a Likert scale, with significantly more women in the TMH cohort indicating that their midwife had no influence on their decision (39\% vs $23 \%$ for PMU cohort) ${ }^{16}$ Similar proportions of each cohort identified their midwives as having 'a lot' of influence $(26 \%$ and $25 \%){ }^{16}$

It is unclear the extent to which the different beliefs and values held by midwives and/or women influence the clinical outcomes in respective birth environments. Research to date has also identified other variables, including the design of a 'birth space,21 22 and the institutional control exerted. ${ }^{23}$ It is difficult to control for these influences, both independently and collectively, and therefore identify the extent of their impact.

The study's strengths include its context of New Zealand's unique system of maternity care, where women receive continuity of care from a midwife, even in the event of specialist consultation and regardless of the place of birth. (Most other studies of PMUs and TMHs are confounded by having to also compare, and not be able to control for, different models of care. ${ }^{6-810-14}$ ) Data were collected prospectively and comprehensively on all aspects of antenatal, labour and postnatal transfers for women planning a primary unit birth in a contemporary western context, where all the women in the study received continuity of care.

Some of the clinical outcomes that were found to be different between primary and tertiary cohorts in other studies were not significantly different here. For example, all other comparable studies have reported higher rates of caesarean section for TMH groups compared with PMU groups, whereas this study found no difference in caesarean section rates-elective or emergency. ${ }^{6-9} 25$ The other New Zealand research had the highest caesarean section rate differential reported to date (relative risk 4.62). ${ }^{9}$ This difference may be due to the small size of the current study or by differences in the timing of the identification of the cohorts. The retrospective Davis et at (2011) study is the only one to identify the cohorts by intended birthplace in labour, whereas the cohorts in each of the other studies were established antenatally (on hospital booking or study entry). Our prospective study found 107 (26.3\%) of the total PMU cohort of 407 women changed plans to give birth at the tertiary hospital prior to labour. The risk status of women at the start of labour cannot be identified for this or the other New Zealand research.

This study, along with the Danish study ${ }^{8}$ found no significant differences in the neonatal outcomes measured. In contrast, the large Birthplace in England study, the Australian EMU study and the previous New Zealand study all reported higher rates of neonatal unit admission for tertiary than primary cohorts. ${ }^{6926}$ What is consistent in these studies is that those who plan to give birth in a freestanding PMU have been shown to have similar or better neonatal outcomes, and none which are worse than the women (of similar risk status) who plan to give birth in a hospital. This study is no exception, further underlying the beneficial results of primary maternity unit care with a higher proportion of Māori women and rural dwelling women (table 1).

The international evidence published to date is that well women who plan to give birth in a TMH are more likely to suffer physical harm in the form of peripartum morbidity, such as episiotomy and caesarean section, than those who plan to give birth in a freestanding PMU. ${ }^{69925-27}$ This morbidity is not resulting in better outcomes for their babies, as the evidence to date, including this study, reports the clinical outcomes for babies from the two groups as either comparable ${ }^{826}$ or better for the PMU cohorts. ${ }^{6} 91128$ The notion that the 'interventions' undertaken at the tertiary hospital on well women are either protecting the wellbeing of women or 'saving babies' is not supported by the evidence published in comparable contexts. Given the complexity of birth, it is difficult to identify causal factors for the increased rate of 'interventions' and their associated morbidities in tertiary when compared with primary facilities. Arguably, it is not possible to undertake a comprehensive and sufficiently powered study to establish the relative physical safety of 
freestanding maternity units and tertiary hospitals for well women at 'low risk' of complications. Birthplace research is difficult and complex, with several potential biases and confounding factors. ${ }^{29-31}$ The commonly held anecdotal association of hospitalisation and reduced mortality and morbidity rates has never been supported by evidence. ${ }^{32}{ }^{33}$ Despite this, the supposition of 'safety' of hospital birth for all women remains, arguably as a result of the power of obstetrics and its hold on 'authoritative knowledge ${ }^{34}{ }^{34}$ Hospital has become the cultural 'gold standard' by default.

While it is possible that rare and severe incidents might happen at a PMU, which might have been prevented or mitigated if the woman was at a TMH, it is also possible that as many iatrogenic or nosocomial rare and severe incidents happen at a TMH. Childbearing women and their families are concerned that their 'safety' is optimised and harm minimised; maternity care providers, healthcare funders and planners share these concerns. This study, and other recent research, has found planning to give birth at a PMU for well ('low risk') women is 'safer' for women and at least as safe for their babies, if safety is defined as 'the reduction of risk of unnecessary harm to an acceptable minimum'. ${ }^{35}$ Even using the narrow outcome measure of physical 'safety' or well-being, the existing evidence does not support hospitals as safer places for well women to give birth, in the context of professional skilled caregivers, effective referral and transfer systems and access to specialist facilities and services for those in need of them.

This study provides clinicians and maternity policy-makers and planners with evidence which supports the safety of freestanding PMUs and their role in a modern integrated maternity system. It illustrates the efficacy of referral and booking guidelines and midwives' use of them in practice. It provides evidence of the effective systems of collaborative multidisciplinary consultation and transfer between facilities, including emergency and non-emergency transport. Generalisation of these findings should be undertaken with caution, as it is unclear if the model of maternity care, with continuity of midwifery care for all participants, has impacted on the outcomes.

Future research, including a larger national prospective cohort study in Aotearoa/New Zealand, would provide stronger evidence of comparative birthplace clinical outcomes between primary and tertiary level facilities in this context. The inclusion of all participants' risk status on admission in labour and the details of antenatal and preadmission labour birthplace plan changes and labour transfers would strengthen the findings. Further research into other potentially influential variables such as the beliefs and values of women and caregivers, birth space design and institutional control would also be valuable in helping to identify the causes of different outcomes for well women giving birth in different context.

\section{CONCLUSION}

This research adds to the growing international body of research on freestanding PMUs. It adds to the worldwide body of research confirming primary units as physically, socially and emotionally/psychologically safe places for well women to plan to give birth, in contexts of maternity systems with integrated midwifery care and primary level maternity units or birth centres. The task now is to disseminate this research, combined with the international primary maternity unit birthplace literature, to childbearing women, maternity care providers and healthcare policy-makers and planners. This research is unique as it is the first birthplace prospective cohort study undertaken in Aotearoa/New Zealand, the only country in the world to have continuity of care as a core tenet in its maternity system. As such, it is important for all those with an interest in all aspects of the safety of birth.

Acknowledgements We thank the women who took part in the study, despite the earthquakes. We also thank the midwives who participated and supported the project, all those who assisted from the MMPO and the participating hospitals and maternity units in Christchurch.

Contributors CPG: principal author; led the New Zealand arm of the study, including its design, recruitment, data collection, cleaning, analysis and interpretation; preparation and modification of the manuscript and carried out all practical aspects of the publication process. SKT: conceived of the study, led its design and coordination; involved in data analysis and interpretation and critical manuscript revision. MT: involved in the design of the study, led data analysis and interpretation; critical manuscript revision and approved the final manuscript. RD: involved in the design and conduct of the study, data interpretation, critical manuscript revision and approved the final manuscript. MK: involved in the design and conduct of the study, critical manuscript revision and approved the final manuscript. AM: involved in the design of the study, coordinated the Australian arm of the EMU project, data interpretation and critical manuscript revision and approved the final manuscript. VS: involved in the conduct of the study, data interpretation, provided critical review and approved the final manuscript.

Funding This study was supported by the National Health and Medical Research Council of Australia (Project Grant number: 571901).

\section{Competing interests None declared.}

Ethics approval New Zealand Upper South B Regional Ethics Committee (URB/09/12/063), 08/02/10. Northern Sydney Health Human Research Ethics Committee (HREC/09/HNE/78); University of Technology Sydney Human Research Ethics Committee; University of Sydney Human Research Ethics Committee (SSA/09/ HNE/79); Hunter New England Human Research Ethics Committee (09/03/18/5.06).

Provenance and peer review Not commissioned; externally peer reviewed.

Data sharing statement No further unpublished data from the study are available.

Open Access This is an Open Access article distributed in accordance with the Creative Commons Attribution Non Commercial (CC BY-NC 4.0) license, which permits others to distribute, remix, adapt, build upon this work non-commercially, and license their derivative works on different terms, provided the original work is properly cited and the use is non-commercial. See: http://creativecommons.org/ licenses/by-nc/4.0/

(c) Article author(s) (or their employer(s) unless otherwise stated in the text of the article) 2017. All rights reserved. No commercial use is permitted unless otherwise expressly granted.

\section{REFERENCES}

1. Ministry of Health. Mo H, ed. New Zealand maternity clinical indicators 2012. Wellington: Ministry of Health, 2014:88.

2. Ministry of Health. Report on Maternity 2014. Wellington, New Zealand: Ministry of Health, 2015. 
3. Ministry of Health. Maternity Services Notice pursuant to section 88 of the New Zealand Public Health and Disability Act 2000. Wellington: Ministry of Health, 2007.

4. Lumley J. The safety of small maternity hospitals in Victoria 1982-84. Community Health Stud 1988;12:386-93.

5. Rosenblatt RA, Reinken J, Shoemack P. Is obstetrics safe in small hospitals? evidence from New Zealand's regionalised perinatal system. Lancet 1985;2:429-23.

6. Monk A, Tracy M, Foureur M, et al. Evaluating Midwifery Units (EMU): a prospective cohort study of freestanding midwifery units in New South Wales, Australia. BMJ Open 2014;4:e006252-11.

7. Brocklehurst $P$, Hardy $P$, Hollowell $\mathrm{J}$, et al. Perinatal and maternal outcomes by planned place of birth for healthy women with low risk pregnancies: the Birthplace in England National Prospective Cohort Study. BMJ 2011:343:1-13.

8. Overgaard C, Møller AM, Fenger-Grøn M, et al. Freestanding midwifery unit versus obstetric unit: a matched cohort study of outcomes in low-risk women. BMJ Open 2011;1:e000262-11.

9. Davis D, Baddock S, Pairman S, et al. Planned place of birth in New Zealand: does it affect mode of birth and intervention rates among low-risk women? Birth 2011;38:111-9.

10. Grunebaum A, McCullough LB, Sapra KJ, et al. Early and total neonatal mortality in relation to birth setting in?the United States, 2006-2009. Am J Obstet Gynecol 2014;211:390.e1-390.e7.

11. Homer CS, Thornton C, Scarf VL, et al. Birthplace in New South Wales, Australia: an analysis of perinatal outcomes using routinely collected data. BMC Pregnancy Childbirth 2014;14:12.

12. Grunebaum A, McCullough LB, Sapra KJ, et al. Apgar score of 0 at 5 minutes and neonatal seizures or serious neurologic dysfunction in relation to birth setting. Am J Obstet Gynecol 2013;209:323.e1-323. e6.

13. Wax JR, Pinette MG, Cartin A, et al. Maternal and newborn morbidity by birth facility among selected United States 2006 low-risk births. Am J Obstet Gynecol 2010;202:152.e1-152.e5.

14. Tracy SK, Dahlen H, Caplice S, et al. Birth centers in Australia: a national population-based study of perinatal mortality associated with giving birth in a birth center. Birth 2007;34:194-201.

15. Grigg CP, Tracy SK, Tracy M, et al. Transfer from primary maternity unit to tertiary hospital in New Zealand - timing, frequency, reasons, urgency and outcomes: part of the evaluating maternity units study. Midwifery 2015;31:879-87.

16. Grigg C, Tracy SK, Daellenbach R, et al. An exploration of influences on women's birthplace decision-making in New Zealand: a mixed methods prospective cohort within the Evaluating Maternity Units study. BMC Pregnancy Childbirth 2014;14:14:14.

17. Ministry of Health. Report on Maternity 2010. Wellington: Ministry of Health, 2012.

18. Grigg CP, Tracy SK, Schmied V, et al. Women's birthplace decisionmaking, the role of confidence: part of the evaluating maternity units study, New Zealand. Midwifery 2015;31:597-605.
19. Miller S, Skinner J. Are first-time mothers who plan home birth more likely to receive evidence-based care? A comparative study of home and hospital care provided by the same midwives. Birth 2012;39:135-44.

20. Davis DL, Walker K. Case-loading midwifery in New Zealand: making space for childbirth. Midwifery 2010;26:603-8.

21. Hammond A, Foureur M, Homer CS, et al. Space, place and the midwife: exploring the relationship between the birth environment, neurobiology and midwifery practice. Women Birth 2013;26:277-81

22. Fahy K, Foureur M, Hastie C. Birth territory and midwifery guardianship: theory for practice, education and research. Elsevier Science: Oxford, 2008.

23. Davis D, Walker K. Towards an ?optics of power?: technologies of surveillance and discipline and case-loading midwifery practice in New Zealand. Gender, Place \& Culture 2013;20:597-612.

24. Seibold C, Licqurish S, et al. 'Lending the space': midwives' perceptions of birth space and clinical risk management. Midwifery 2010;26:526-31.

25. Walsh D, Downe SM. Outcomes of free-standing, midwife-led birth centers: a structured review. Birth 2004;31:222-9.

26. Hollowell J, Puddicombe D, Rowe R, et al. The Birthplace nationalprospective cohort study: perinatal and maternal outcomes by planned place of birth. Birth PlaceEngland research programme. Final report part 4. National Perinatal Epidemiology Unit, Oxford, UK NIHR Service Delivery and Organisation programme, 2011.

27. Tew M. Obstetric hospitals and general-practitioner maternity units-the statistical record. J R Coll Gen Pract 1977;27:689-94.

28. Laws PJ, Tracy SK, Sullivan EA. Perinatal outcomes of women intending to give birth in birth centers in Australia. Birth 2010;37:28-36.

29. de Vries RG, Paruchuri Y, Lorenz K, et al. Moral science: ethical argument and the production of knowledge about place of birth. $J$ Clin Ethics 2013;24:225-38.

30. Nove A, Berrington A, Matthews Z. The methodological challenges of attempting to compare the safety of home and hospital birth in terms of the risk of perinatal death. Midwifery 2012;28:619-26.

31. Keirse MJ. Home birth: gone away, gone astray, and here to stay. Birth 2010;37:341-6.

32. Tew M. Safer childbirth?: a critical history of maternity care. Oxford, UK: Oxford University Press, 1990.

33. Donley J. Birthrites, Natural vs unnatural childbirth in New Zealand. Auckland, New Zealand: The Full Court Press, 1998.

34. Jordan B. Authoritative knowledge and its construction. In: DavisFloyd R, Sargent C, eds. Childbirth and authoritative knowledge. Berkley, California: University of California Press, 1997:55-79.

35. Runciman W, Hibbert P, Thomson R, et al. Towards an International Classification for Patient Safety: key concepts and terms. Int J Qual Health Care 2009;21:18-26. 